\title{
Changes on Confocal Scanning Laser Ophthalmoscopy with the Heidelberg Retinal Tomography after a Cardiac Catheterism in a Patient with Progressive Glaucoma
}

\author{
Diego Alejandro Valera-Cornejo ${ }^{a, d}$ Waldo Loayza-Gamboa ${ }^{a, b, d}$ \\ Julio Herrera-Quiroz ${ }^{a, d}$ Rosa Alvarado-Villacorta ${ }^{a, c, d}$ \\ Luis Córdova-Crisanto ${ }^{a, e}$ Vanessa Valderrama-Albino ${ }^{a, b}, d$ \\ Nahuel Pantoja-Dávalos ${ }^{a, b}$ e

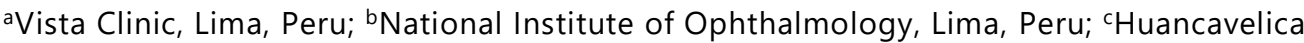 \\ General Hospital, Huancavelica, Peru; ${ }^{d}$ Cayetano Heredia National University, Lima, Peru; \\ eSan Marcos National University, Lima, Peru
}

\section{Keywords}

Open-angle glaucoma $\cdot$ Heidelberg retinal tomography $3 \cdot$ Blood flow $\cdot$ Imaging technique .

Disease progression

\begin{abstract}
Purpose: We present a case of a patient with progressive open angle glaucoma who presented changes suggestive of improvement in the Heidelberg retinal tomography 3 (HRT3) analysis after a cardiac catheterization. Observation: A 69-year-old woman presented with progressive open angle glaucoma despite maximum tolerable antiglaucomatous topical treatment. A filtering surgery (trabeculectomy) was performed and successfully achieved intraocular pressure (IOP) levels of $10 \mathrm{~mm} \mathrm{Hg}$ on average. Despite this, changes were evidenced in the HRT3 protocols (trend analysis and topographic change analysis) suggesting marked progression. Brimonidine $0.2 \%$ twice a day was initiated, and a cardiovascular examination was requested. A cardiac catheterism was performed in the following weeks, and afterward, all structural
\end{abstract}


Valera-Cornejo et al.: Changes on Confocal Scanning Laser Ophthalmoscopy with the Heidelberg Retinal Tomography after a Cardiac Catheterism in a Patient with Progressive Glaucoma

parameters improved until the last control. Medication was not discontinued, and no signs of apparent progression on the HRT3 parameters have been evidenced up until the time of writing this case report. Conclusions and Importance: There was a marked improvement in the HRT3 parameters (trend and topographic change analysis), suggesting that the progression stopped after a cardiac catheterism in a patient with progressive glaucoma despite having the IOP controlled. To our knowledge, this is the first case of a patient with progressive glaucoma that was medically and surgically managed, and despite achieving low IOP levels, the progression detected by the HRT3 analysis could not be stopped until a cardiac catheterization was performed.

\section{Introduction}

The etiology of primary open-angle glaucoma it still not well understood, despite all the extensive research available. Glaucoma is a multifactorial disease, and modifying only one factor (intraocular pressure - IOP) limits our treatment. We know that the IOP is the most important risk factor, but it is known that the pressure does not necessarily have to be high for glaucoma to progress. A mechanism of disease causation would relate to alterations of ocular perfusion pressure [1], leading to glaucomatous damage of the optic nerve head (ONH).

In many studies, cardiovascular disease (CVD) was evidenced as a risk factor, even regardless of IOP [2-4]. This reinforces the importance of the vascular component in the pathogenesis of glaucoma (vascular theory). This theory sustains that glaucoma is caused by decreased ocular perfusion pressure, which affects ganglion cells at the $\mathrm{ONH}$, and disease progression is enhanced by this vascular dysregulation [5]. Systemic hypotension, nocturnal blood pressure dipping, low diastolic perfusion pressure, and low ocular perfusion pressure has also been implicated with rapid disease progressors, especially in normal tension glaucoma (NTG) [6,7]. Vascular diseases such as diabetes and CVD have not been consistently shown to be correlated with progression $[3,4]$.

Objective technologies such as the Heidelberg retinal tomography (HRT; Heidelberg Engineering, $\mathrm{GmbH}$, Dossenheim, Germany) have been developed as adjuncts to subjective $\mathrm{ONH}$ evaluation. The HRT is a confocal scanning laser tomography device that creates reproducible and repeatable three-dimensional topographic images of the $\mathrm{ONH}$ and the peripapillary retina and had a different software that can aid evaluate progression through time, such as the topographic change (TCA) and trend analysis [8]. There is increasing evidence in the literature that TCA and trend analysis can detect progressive optic disc changes [9-12].

The present report shows us the importance of these vascular factors and how treating one of them can change the apparent structural worsening of the disease in our patient with glaucoma.

\section{Case Report}

\section{Clinical History}

A 69-year-old female with a single eye (history of retinal detachment surgery of the contralateral eye) and primary open-angle glaucoma treated with maximum medical topical therapy (timolol $0.5 \%$, dorzolamide $2 \%$, brimonidine $0.2 \%$, and latanoprost $0.005 \%$ ) presented at our clinic. Medical history included essential arterial hypertension, coronary insufficiency and episodes of angina pectoris. 
Clinical findings were the following: central corneal thickness of $504 \mu \mathrm{m}$, vertical relation cup-to-disc ratio of 0.88, Shaffer gonioscopy of IV in three quadrants (with little pigment), crystalline opacity (LOCS III) NO1NC1C0P0 with basal examinations (visual field [VF] and HRT3) shown in Figure 1 and 2.

Despite maximally tolerable antiglaucomatous topical treatment, intraocular pressure ranged between $18 \mathrm{~mm} \mathrm{Hg}$ and $20 \mathrm{~mm} \mathrm{Hg}$. During the follow-up between 2013 and 2014 (8 months), she presented signs of progression in the trend analysis of HRT3 (Fig. 3), and it was decided then to perform a filtering surgery (trabeculectomy). The procedure was done without complications achieving IOP levels of $13 \mathrm{~mm} \mathrm{Hg}$ on average, with a functional filtering bleb and without additional medical therapy.

However, 20 months later, the persistence of progression in the trend analysis and topographic analysis of changes (TCA) of HRT3 was evidenced (Fig. 3 and 4). During this time, she maintained a mean IOP of $11 \mathrm{~mm} \mathrm{Hg}$ throughout the follow-up. At this point, the problem was "What else can be done to halt this apparent progression in a patient with a single functional eye, if the pressure is controlled?" It was then decided to use medical treatment with brimonidine $0.20 \%$ twice a day, and a cardiological evaluation was also requested.

The following month, the cardiology department from a local hospital performed two cardiac catheterizations, after which the HRT3 parameters improved considerably (Fig. 3 and 4). After 11 months, there is still improvement in HRT3 parameters; currently, the patient uses brimonidine $0.20 \%$ b.i.d. and has IOPs of $14 \mathrm{~mm} \mathrm{Hg}$. On average, VF examinations did not show any change throughout the follow-up (Fig. 5).

\section{Discussion}

The HRT system allows us to evaluate the change over time with two protocols: trend analysis map and TCA $[8,9]$. The TCA map is an event-based protocol for detecting topographic surface height changes across the entire $\mathrm{ONH}$ and peripapillary surface at a superpixel level between baseline and follow-up images. Progression is identified when the change exceeds measurement variability and is confirmed in, at least, 3 consecutive tests (error probability $<5 \%$ ) and a cluster of 20 or more significantly depressed superpixels [9].

We have to remember that when talking about progression we cannot rely only on one test; nevertheless, some studies have demonstrated that there is a significant subset of patients who show TCA changes without ONH stereophotograph [9-11] or VF progression as was probably the case in our report. Like our case, some patients with open-angle glaucoma continue to progress despite an adequate decrease in the IOP. We know that there are several risk factors besides IOP, such as a thin central corneal thickness, pseudo-exfoliation, and other vascular entities like systemic hypotension, that have also been implicated in the progression and pathogenesis of glaucoma; this particular type of glaucoma is known as NTG and is explained by the "vascular theory" [13]. These patients have a low perfusion pressure of the ONH caused by an altered vascular regulation that eventually leads to unstable ocular perfusion, ischemia, and even damage by reperfusion of the head of the optic nerve [14].

There is evidence to suggest that low and fluctuating perfusion pressure are risk factors for the development of glaucoma [15]; reduced ocular blood flow is also associated with its progression and pathogenesis [16]. Patients with diabetes and arteriosclerosis are supposed to have reduced retinal blood flow due to the endothelial and capillary dysfunction from the microvascular damage of the disease, and this is evidenced by large clinical studies, which found a higher prevalence of glaucoma in these patients even independent from IOP $[17,18]$. 
But ocular blood flow is not as simple as it looks, because the vascular factors related in the pathogenesis of NTG are unclear; oxidative stress, vasospasm, and endothelial dysfunction seem likely to be the cause of glaucomatous optic neuropathy.

CVD is an important risk factor for progression of glaucoma, perhaps independently of IOP [2]. Chan et al. [2] found that the progression of glaucoma was more rapid in patients with CVD, and these patients doubled the possibility of disease progression. Patients who had CVD in the rapid progression group of this study had lower IOP than controls, indicating that this could be an independent factor. Another study based on the Early Manifest Glaucoma Trial (EMGT) found that patients with CVD had a hazard ratio of 2.75 for glaucoma disease progression compared with non-CVD patients $[3,4]$.

This could explain the reason why our patient had a consistent change in the topographic and trend maps that suggested progression despite having good IOP levels (achieved with surgery and medication), and these changes improved only after a cardiac catheterization, suggesting that the structural progression stopped and even improved. One possible additional factor influencing this improvement could be the addition of brimonidine at that moment, but IOP levels were already low during the follow-up, and its neuroprotective mechanism or its additional effect on the pressure could probably explain this improvement.

We could not find any similar cases in the literature, so we believe it is a valuable report. The prevalence and effects of CVD in patients with glaucoma are well known from the large population trials published in the literature [19-23], but how these diseases relate to more aggressive progression, and which ones in particular, is not known yet. On the other hand, a few studies showed no association between CVD history and glaucoma progression [24, 25].

Thus, decreasing only the IOP for the treatment of glaucoma is not enough. A complete medical history is of tremendous importance in our patients with glaucoma, especially those with CVDs. The aim is to try to modify a risk factor that could decrease progression in these patients, and the therapeutic strategy should also include neuroprotection. If we can modify a risk factor, then it should be treated.

\section{Acknowledgments}

We gratefully thank the patient and her family for participation in this study.

\section{Statement of Ethics}

Informed written consent was obtained from the patient for publication of personal and medical record details.

\section{Disclosure Statement}

The authors have no financial disclosures or conflict of interest.

\section{Funding Sources}

No funding or grant support. 


\section{References}

1 Nicolela MT. Clinical clues of vascular dysregulation and its association with glaucoma. Can J Ophthalmol. 2008 Jun;43(3):337-41.

2 Chan TC, Bala C, Siu A, Wan F, White A. Risk Factors for Rapid Glaucoma Disease Progression. Am J Ophthalmol. 2017 Aug;180:151-7.

3 Leske MC, Heijl A, Hyman L, Bengtsson B, Dong L, Yang Z; EMGT Group. Predictors of long-term progression in the early manifest glaucoma trial. Ophthalmology. 2007 Nov;114(11):1965-72.

4 Leske MC, Heijl A, Hussein M, Bengtsson B, Hyman L, Komaroff E, et al. Factors for glaucoma progression and the effect of treatment: the early manifest glaucoma trial. Arch Ophthalmol. 2003 Jan;121(1):48-56.

5 Cherecheanu AP, Garhofer G, Schmidl D, Werkmeister R, Schmetterer L. Ocular perfusion pressure and ocular blood flow in glaucoma. Curr Opin Pharmacol. 2013 Feb;13(1):36-42.

6 Charlson ME, de Moraes CG, Link A, Wells MT, Harmon G, Peterson JC, et al. Nocturnal systemic hypotension increases the risk of glaucoma progression. Ophthalmology. 2014 Oct;121(10):2004-12.

7 Krupin T, Liebmann JM, Greenfield DS, Rosenberg LF, Ritch R, Yang JW; Low-Pressure Glaucoma Study Group. The Low-pressure Glaucoma Treatment Study (LoGTS) study design and baseline characteristics of enrolled patients. Ophthalmology. 2005 Mar;112(3):376-85.

8 Chauhan BC, Blanchard JW, Hamilton DC, LeBlanc RP. Technique for detecting serial topographic changes in the optic disc and peripapillary retina using scanning laser tomography. Invest Ophthalmol Vis Sci. 2000 Mar;41(3):775-82.

9 Chauhan BC, Hutchison DM, Artes PH, Caprioli J, Jonas JB, LeBlanc RP, et al. Optic disc progression in glaucoma: comparison of confocal scanning laser tomography to optic disc photographs in a prospective study. Invest Ophthalmol Vis Sci. 2009 Apr;50(4):1682-91.

10 Kourkoutas D, Buys YM, Flanagan JG, Hatch WV, Balian C, Trope GE. Comparison of glaucoma progression evaluated with Heidelberg retina tomograph II versus optic nerve head stereophotographs. Can J Ophthalmol. 2007 Feb;42(1):82-8.

11 Bowd C, Balasubramanian M, Weinreb RN, Vizzeri G, Alencar LM, O'Leary N, et al. Performance of confocal scanning laser tomograph Topographic Change Analysis (TCA) for assessing glaucomatous progression. Invest Ophthalmol Vis Sci. 2009 Feb;50(2):691-701.

12 Ervin JC, Lemij HG, Mills RP, Quigley HA, Thompson HW, Burgoyne CF. Clinician change detection viewing longitudinal stereophotographs compared to confocal scanning laser tomography in the LSU Experimental Glaucoma (LEG) Study. Ophthalmology. 2002 Mar;109(3):467-81.

13 Orgul S, Zawinka C, Gugleta K, Flammer J. Therapeutic strategies for normal-tension glaucoma. Ophthalmologica. 2005 Nov-Dec;219(6):317-23.

14 Flammer J, Orgül S, Costa VP, Orzalesi N, Krieglstein GK, Serra LM, et al. The impact of ocular blood flow in glaucoma. Prog Retin Eye Res. 2002 Jul;21(4):359-93.

15 Sung KR, Cho JW, Lee S, Yun SC, Choi J, Na JH, et al. Characteristics of visual field progression in medically treated normal-tension glaucoma patients with unstable ocular perfusion pressure. Invest Ophthalmol Vis Sci. 2011 Feb;52(2):737-43.

16 Satilmis M, Orgül S, Doubler B, Flammer J. Rate of progression of glaucoma correlates with retrobulbar circulation and intraocular pressure. Am J Ophthalmol. 2003 May;135(5):664-9.

17 Klein BE, Klein R, Jensen SC. Open-angle glaucoma and older-onset diabetes. The Beaver Dam Eye Study. Ophthalmology. 1994 Jul;101(7):1173-7.

18 Mitchell P, Smith W, Chey T, Healey PR. Open-angle glaucoma and diabetes: the Blue Mountains eye study, Australia. Ophthalmology. 1997 Apr;104(4):712-8.

19 Mitchell P, Lee AJ, Rochtchina E, Wang JJ. Open-angle glaucoma and systemic hypertension: the blue mountains eye study. J Glaucoma. 2004 Aug;13(4):319-26.

20 Wilson MR, Hertzmark E, Walker AM, Childs-Shaw K, Epstein DL. A case-control study of risk factors in open angle glaucoma. Arch Ophthalmol. 1987 Aug;105(8):1066-71.

21 Memarzadeh F, Ying-Lai M, Chung J, Azen SP, Varma R; Los Angeles Latino Eye Study Group. Blood pressure, perfusion pressure, and open-angle glaucoma: the Los Angeles Latino Eye Study. Invest Ophthalmol Vis Sci. 2010 Jun;51(6):2872-7.

22 Lee AJ, Wang JJ, Kifley A, Mitchell P. Open-angle glaucoma and cardiovascular mortality: the Blue Mountains Eye Study. Ophthalmology. 2006 Jul;113(7):1069-76.

23 Topouzis F, Wilson MR, Harris A, Founti P, Yu F, Anastasopoulos E, et al. Risk factors for primary open-angle glaucoma and pseudoexfoliative glaucoma in the Thessaloniki eye study. Am J Ophthalmol. 2011 Aug;152(2):219-228.e1.

24 Wesselink C, Marcus MW, Jansonius NM. Risk factors for visual field progression in the groningen longitudinal glaucoma study: a comparison of different statistical approaches. J Glaucoma. 2012 Dec;21(9):579-85.

25 Feraru C, Chiseliță D, Pantalon A. Long-term progression and risk factors in primary open-angle glaucoma in clinical care. Spektrum Augenheilkd. 2016 Oct;30(4-5):181-9. 
Valera-Cornejo et al.: Changes on Confocal Scanning Laser Ophthalmoscopy with the Heidelberg Retinal Tomography after a Cardiac Catheterism in a Patient with Progressive Glaucoma

Waldo Loayza-Gamboa, Julio Herrera-Quiroz, Rosa Alvarado-Villacorta, Luis Córdova-Crisanto, Vanessa Valderrama-Albino, Nahuel Pantoja-Davalos are co-authors of this work 


\section{Case Reports in Ophthalmology}

Case Rep Ophthalmol 2019;10:256-266

(c) 2019 The Author(s). Published by S. Karger AG, Basel www.karger.com/cop

Valera-Cornejo et al: Changes on Confocal Scanning Laser Ophthalmoscopy with the Heidelberg Retinal Tomography after a Cardiac Catheterism in a Patient with Progressive Glaucoma

\begin{tabular}{|c|c|c|}
\hline \multicolumn{2}{|c|}{$\begin{array}{l}\text { Heidelberg Retina Tomograph } \\
\text { Regression Analysis }\end{array}$} & 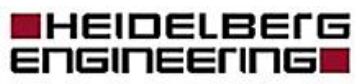 \\
\hline Patlent: & & \\
\hline Examination: & $\begin{array}{l}\text { Sex: female DOB: } 07 / \text { feb/1947 Pat-ID: -- Ethnicity: (Caucasian) } \\
\text { Date: } 19 / \text { mar/2013 }\end{array}$ & \\
\hline Scan: & Focus: $-1.00 \mathrm{dpt}$ Depth: $4.00 \mathrm{~mm}$ Operator: SHIRLEY IOP:-- & \\
\hline
\end{tabular}

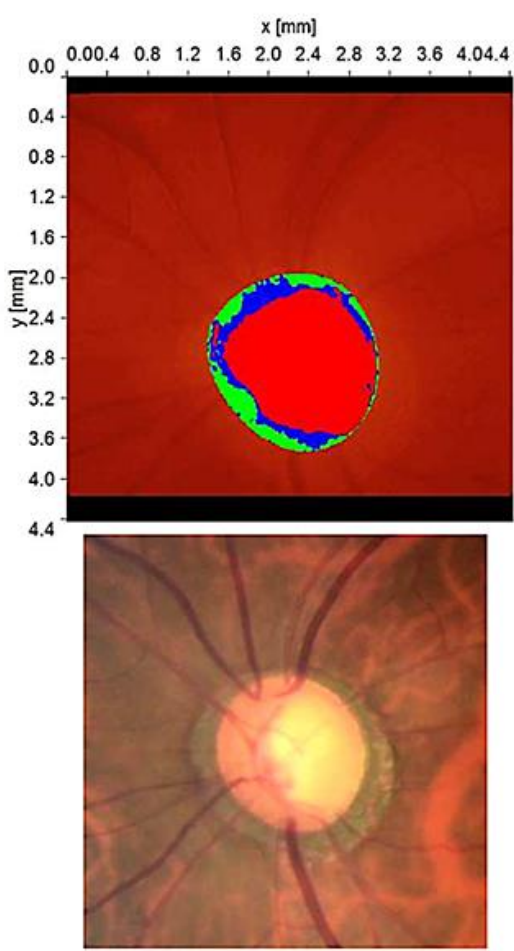

$x[\mathrm{~mm}]$

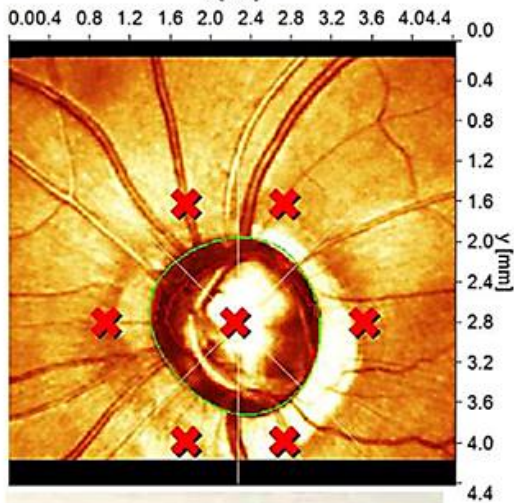

cup

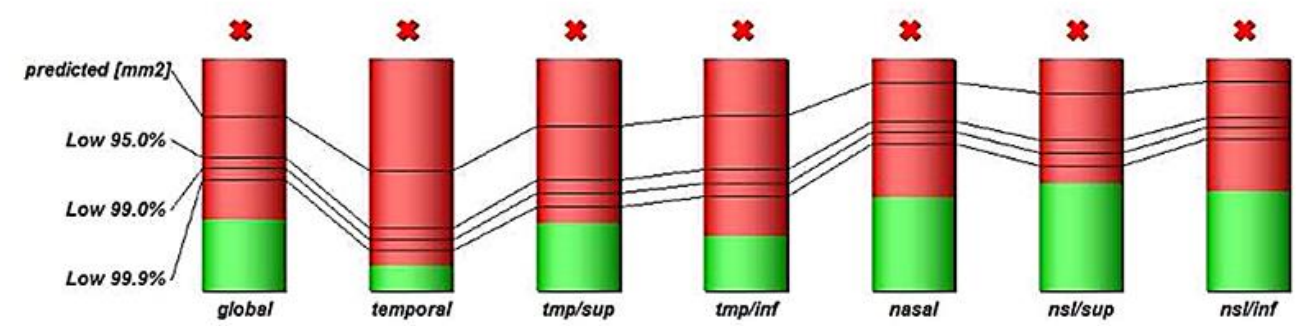

Moorfields Regression Classification: Outside normal limits (")

()) Moorfe'ds regression classiscation (Ophthalmology 1998, 105:1557-1563). Classifcation based on stajstics. Diagnosis is physician's responsibaity.

Fig. 1. Baseline optic disc photograph, visual field, and Heidelberg retina tomography 3 parameters from 2013. Left eye shows diffuse rim thinning, with Moorfields regression classification outside normal limits. 


\section{Case Reports in Ophthalmology}

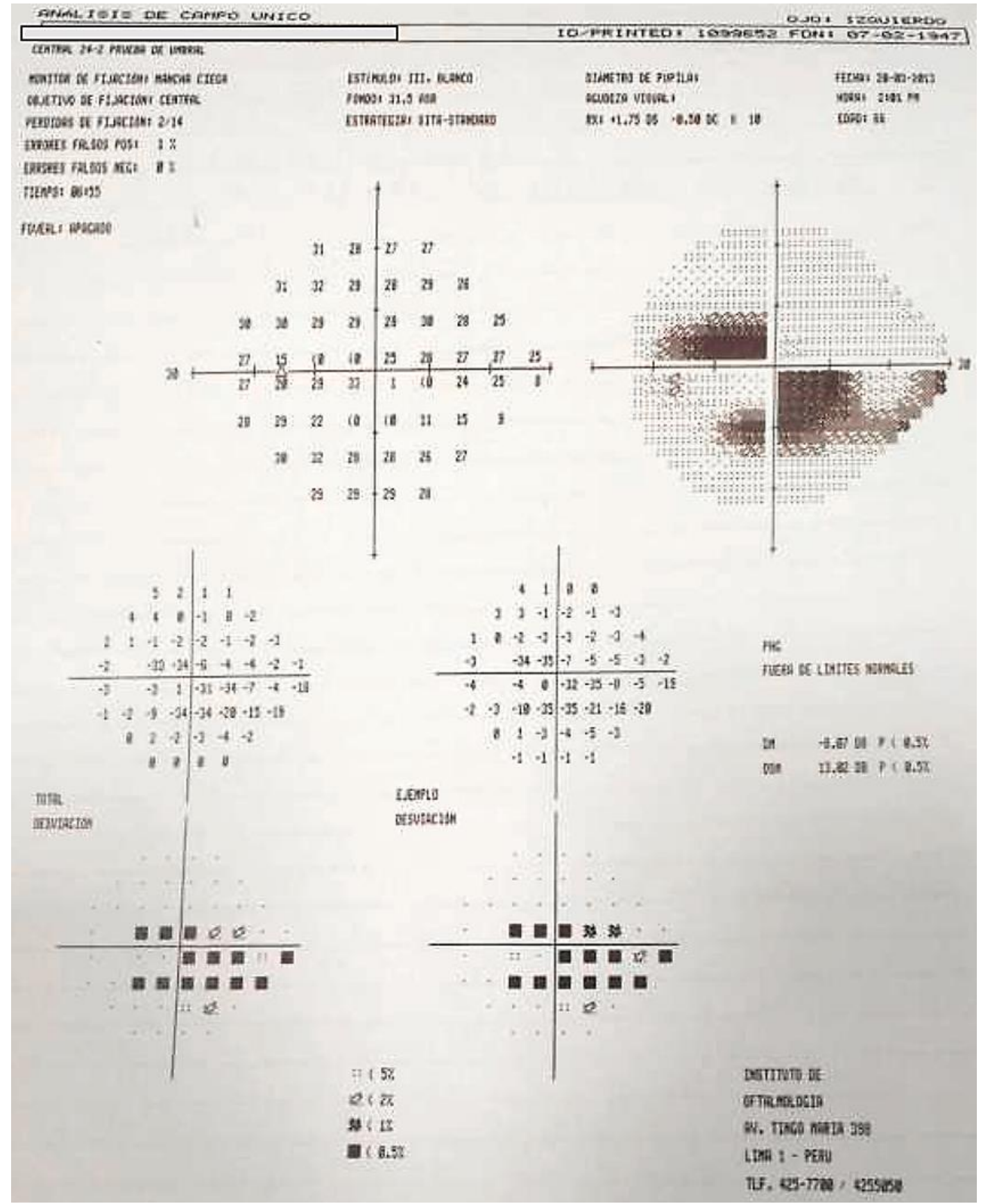

Fig. 2. Humphrey visual field examination from the initial evaluation. Left eye showed good reliability and

a focal (cecocentral, paracentral, and inferior nasal) defect.

Valera-Cornejo et al: Changes on Confocal Scanning Laser Ophthalmoscopy with the Heidelberg Retinal Tomography after a Cardiac Catheterism in a Patient with Progressive Glaucoma 


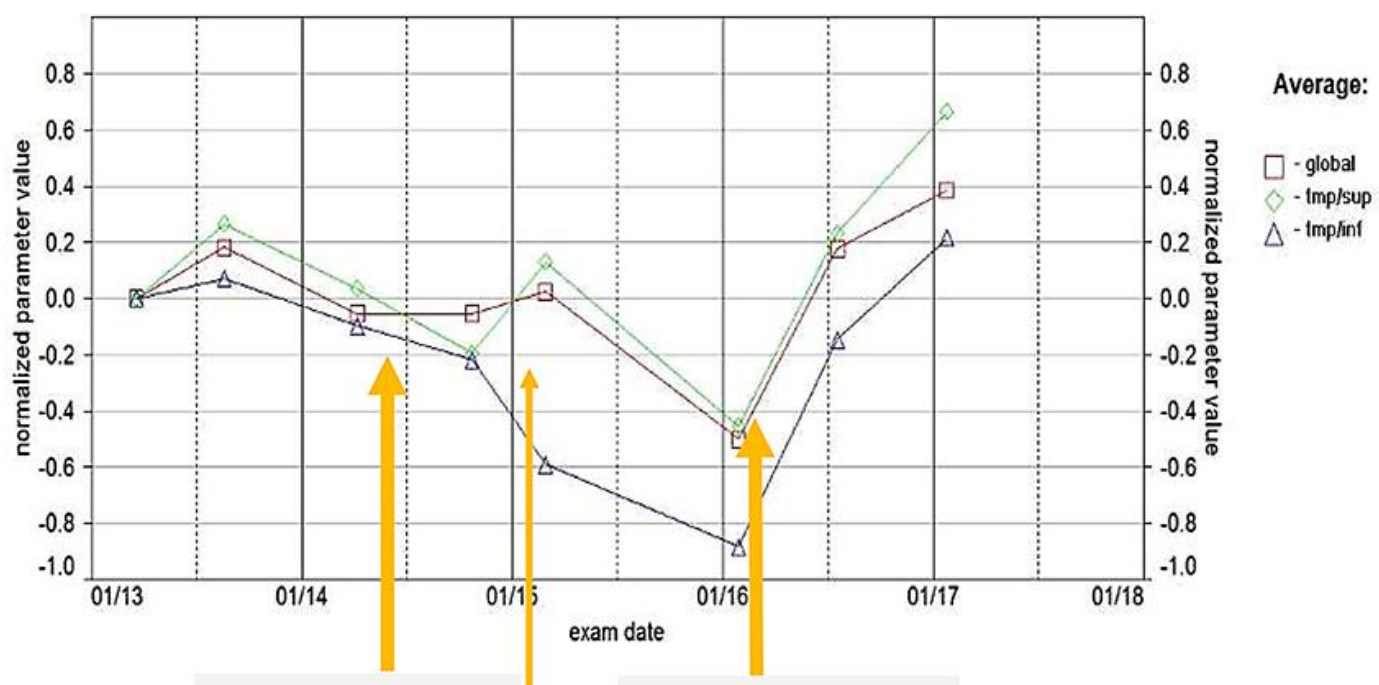

Trabeculectomy

Cardiac catheterism IOP mm Hg $18 \mathrm{~mm} \mathrm{Hg} \quad 12 \mathrm{~mm} \mathrm{Hg} \quad 10 \mathrm{~mm} \mathrm{Hg} \quad 14 \mathrm{~mm} \mathrm{Hg}$

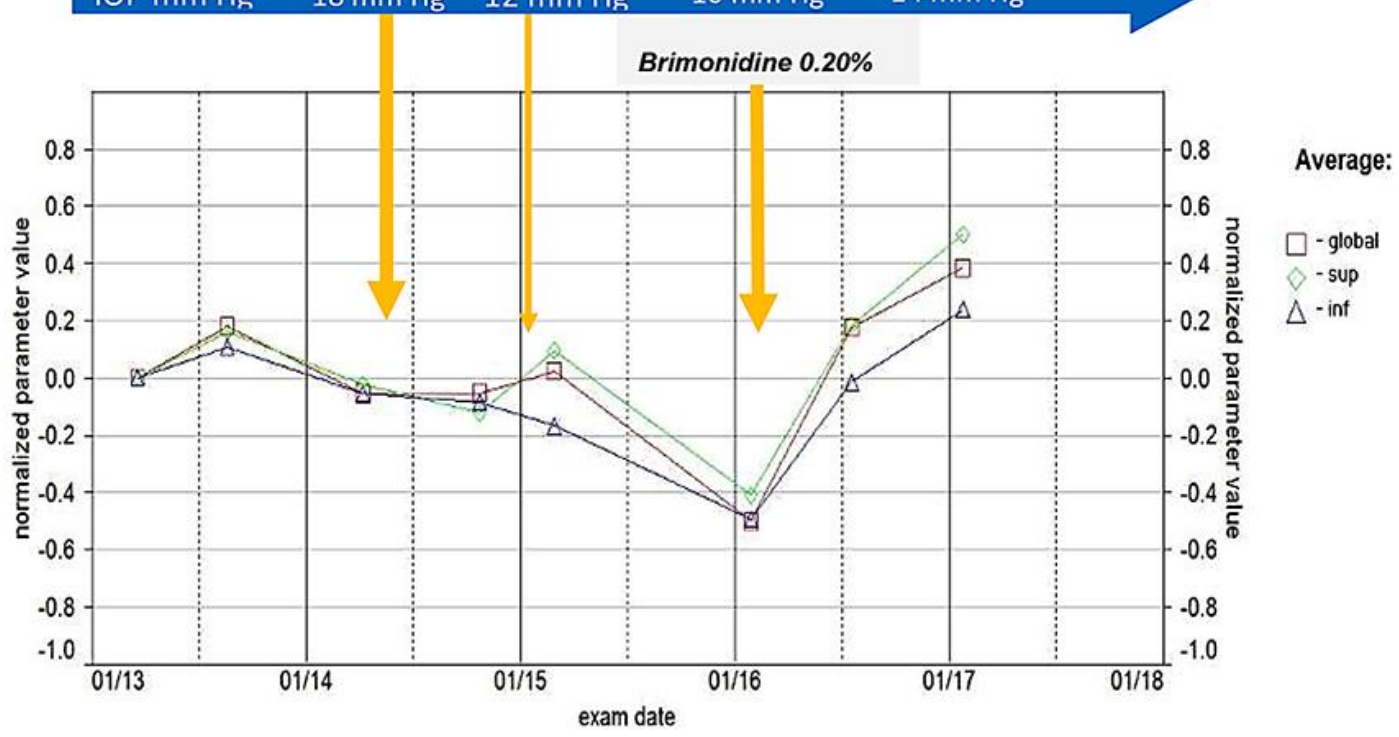

Fig. 3. Heidelberg retinal tomography 3 trend analysis and follow-up. The left eye shows that despite trabeculectomy and apparently good intraocular pressure levels, progression continued, and only after the cardiac catheterism, the parameters improved. 


\section{Case Reports in Ophthalmology}

Heidelberg Retina Tomograph TCA Overview

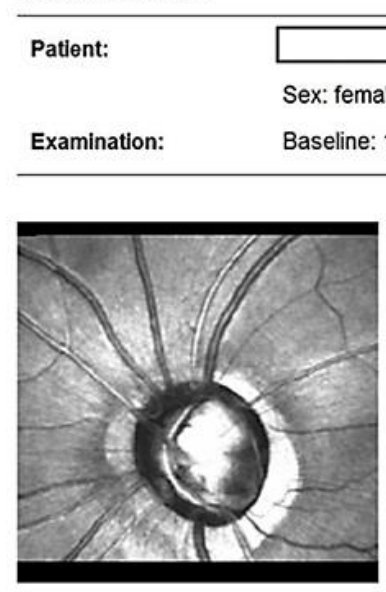

Baseline: 19/mar/2013

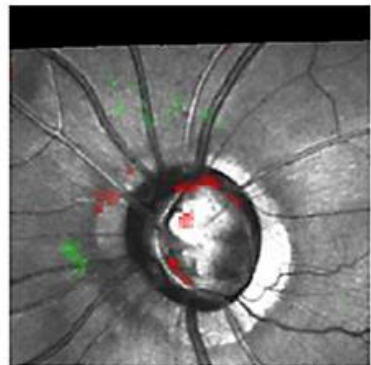

Follow-Up: \#2, 07/abr/2014

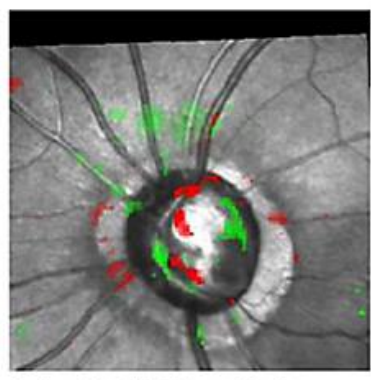

Follow-Up: \#5, 28/ene/2016
Case Rep Ophthalmol 2019;10:256-266

DOI: 10.1159/000501709

(c) 2019 The Author(s). Published by S. Karger AG, Basel www.karger.com/cop

Valera-Cornejo et al: Changes on Confocal Scanning Laser Ophthalmoscopy with the Heidelberg Retinal Tomography after a Cardiac Catheterism in a Patient with Progressive Glaucoma

\section{口HEIDELBETL EПБIПЕЕГІПББ}

ex: female DOB:07/feb/1947 Pat-ID: --- Ethnicity: (Caucasian)

seline: 19/mar/2013 Last Follow-Up: 23/ene/2017 Elapsed: 46 months

OS

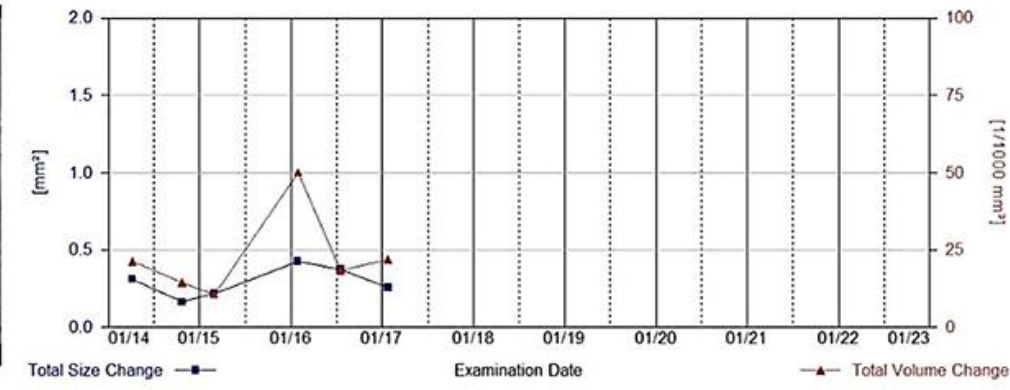

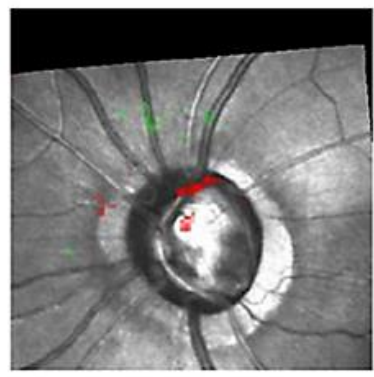

Follow-Up: \#3, 23/oct/2014

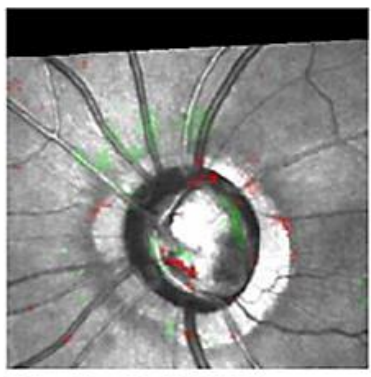

Follow-Up: \#6, 18/ju/2016

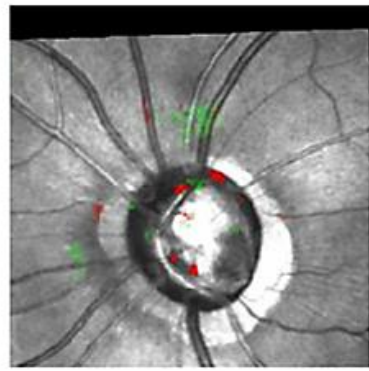

Follow-Up: \#4, 27/feb/2015

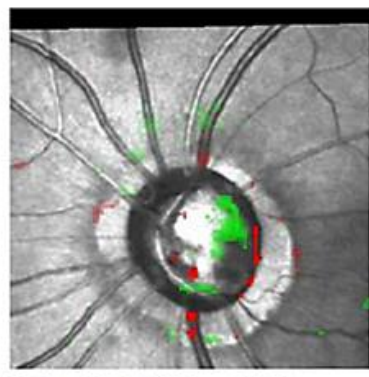

Follow-Up: \#7, 23/ene/2017

Fig. 4. Heidelberg retinal tomography 3. Topographic change analysis during follow-up showing improvement (green areas) after the cardiac procedure. 
Humphrey visual field examination from initial evaluation (2013)
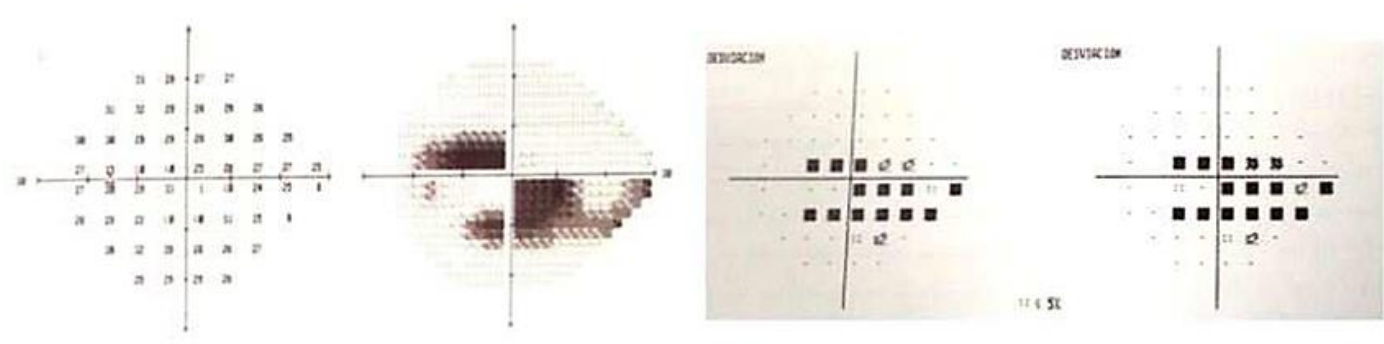

Humphrey visual field examination after cardiac catheterism (2016)
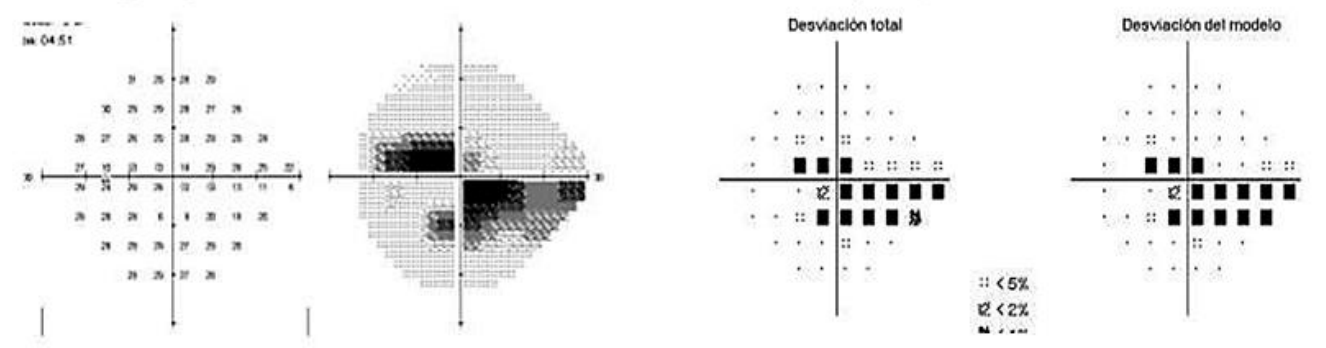

Fig. 5. Humphrey visual field examination from the initial evaluation (2013 and after the cardiac procedure) showing the same focal defect with no changes. 